\title{
Comparação entre métodos químicos de controle de buva com início de dessecação em período seco ou úmido
}

\author{
Comparison of horseweed chemical control methods with burndown \\ starting in dry or moist period
}

Caio Vitagliano Santi Rossi ${ }^{1 *}$, Luiz Henrique Saes Zobiole ${ }^{1}$, Felipe Ridolfo Lucio ${ }^{1}$, Rodrigo Neves $^{1}$

Resumo - Diversas são as ocasiões onde o clima interfere diretamente na eficácia dos herbicidas aplicados para o controle de plantas daninhas. Dessa forma, objetivou-se com este trabalho avaliar o melhor momento climático para iniciar o controle químico da buva (Conyza spp.). Os experimentos foram conduzidos no município de Indianópolis/ MG. $\mathrm{O}$ experimento 1 foi realizado em esquema fatorial (2x5), tendo como primeiro fator as condições climáticas, período seco ou úmido, no momento da primeira aplicação do herbicida glifosato $+2,4-\mathrm{D}(1.200+670$ g e.a. ha-1 $)$, e o segundo fator a aplicação sequencial representada pelos tratamentos herbicidas (g e.a ou i.a. ha-1): glifosato + 2,4-D (1.200+670) ou paraquat (500), isolados ou associados ao diclosulam $(29,4)$, fluroxipir (200) isolado, além da testemunha absoluta sem aplicação de herbicida. O experimento 2 visou comprovar o nível de dificuldade de controle da buva ao herbicida glifosato na área de ambos experimentos, em aplicações sequenciais, espaçadas 35 dias entre as mesmas, de doses (g e.a ha-1) crescentes de glifosato $(0,480,960,1.920$ e 3.840), 2,4-D (670) e a associação de glifosato + 2,4-D $(960+670)$. De forma geral, para se obter controle efetivo, iniciando a aplicação em período seco ou úmido, foi necessário realizar aplicação sequencial usando na primeira o glifosato +2,4-D seguida de uma segunda aplicação, independente dos herbicidas testados. A população de buva presente na área demonstrou não ser controlada somente com aplicações do herbicida glifosato.

Palavras-chave: clima, herbicida, manejo, planta daninha

Abstract - Several environmental conditions may directly interferes with the effectiveness of herbicides weed control, therefore, the aim of this research was to evaluate the best climate moment to initiate chemical horseweed (Conyza spp.) control; as well as to evaluate the level of glyphosate tolerance on horseweed population. These trials were conducted in Indianópolis region, Minas Gerais State. The experiment 1 was designed as factorial $(2 \times 5)$ with first factor being the weather period conditions, dry or wet, comparison at first application time of glyphosate $+2,4-\mathrm{D}\left(1,200+670 \mathrm{~g}\right.$ a.e. ha $\left.{ }^{-1}\right)$ herbicide. The second factor was the sequential herbicide ( $\mathrm{g}$ a.e. or a.i. ha ${ }^{-1}$ ) application with glyphosate $+2,4-\mathrm{D}$ $(1,200+670)$ or paraquat $(500)$, alone or tank mixed with diclosulam (29.4); fluroxypyr (200) alone and an untreated without herbicide application. The experiment 2 aimed to assess the Conyza spp. difficulty level of control to glyphosate herbicide in both trials area by applying sequential applications, 35 days apart, of glyphosate rates (g a.e. ha ${ }^{-1}$ ) at 0, 480, 960, 1,920 and 3,840 as well as 2,4-D (670) and glyphosate + 2,4-D (960 + 670). In general, to obtain effective control, either in

Recebido: 02 de agosto de 2018. Aceito: 01 de novembro de 2018.

${ }^{1}$ Corteva Agriscience ${ }^{\mathrm{TM}}$ Divisão Agrícola da DowDuPont ${ }^{\mathrm{TM}}$, Rodovia SP 147, Km 71,5, CEP 13800-970, Mogi

Mirim, SP, Brasil. E-mail: cvrossi@dow.com; 1szobiole@dow.com; frlucio@dow.com; rneves@dow.com 
dry or wet conditions at first application, it was necessary to perform sequential application using glyphosate + 2,4-D at first application followed by a second one, independent of tested herbicides. The horseweed population present in the area showed not to be controlled only by glyphosate herbicide applications.

Keywords: weather, herbicide, management, weed

\section{Introdução}

A buva é uma planta daninha habitualmente anual, facultativas de inverno, tendo possibilidade de emergir tanto no outono como na primavera, ou mesmo nas duas temporadas sempre que as condições ambientais sejam as propicias (Cici e Van Acker, 2009; Tozzi e Van Acker, 2014). As principais espécies no Brasil são Conyza canadensis, C. bonariensis e C. sumatrensis; sendo que a primeira se diferencia morfologicamente das outras espécies, no entanto $C$. bonariensis e C. sumatrensis apresentam menor grau de diferenciação entre elas (Theabaud e Abbott, 1995). Porém, a partir de grupos definidos de genomas ancestrais nos locos microssatélites (SSR) para as espécies C. canadensis, $C$. bonariensis e C. sumatrensis, foi possível identificar plantas com o genoma ancestral de $C$. canadenses onde C. sumatrensis era a espécie predominante, além disso, foram detectadas amostras de Conyza com genoma ancestral de duas ou três espécies, tornando-se complicada a identificação de buva a partir de características morfológicas, devido a essa hibridação de espécies que pode ter ocorrido nas lavouras do Sul do Brasil (Marochio et al., 2017).

São espécies muito prolíficas, sendo que a C. bonariensis tem a capacidade de produzir 375.000 sementes por planta, a $C$. canadensis em torno de 200.000 e a $C$. sumatrensis mais de 60.000 (Green, 2010). Ainda há relatos que uma planta de $C$. canadensis pode produzir até 792.000 sementes (Shrestha et al., 2010).

Atualmente, milhões de hectares agrícolas estão infestados por biótipos de buva resistente ao glifosato, principalmente em culturas anuais do
Estado do Rio Grande do Sul com a C. bonariensis (Vargas et al., 2007), em culturas perenes como citros no Estado de São Paulo com a C. bonariensis e C. canadensis (Moreira et al., 2007) e no Estado do Paraná a $C$. bonariensis resistente ao glifosato e a $C$. sumatrensis apresentando resistência múltipla ao glifosato e chlorimuron (Santos et al., 2014; Heap, 2017b). Na região Centro-Oeste do Brasil, as infestações de buva preocupam os produtores dessas áreas agrícolas, pois mesmo em altas doses de glifosato tem se observado ineficiência, sendo um forte indicativo de biótipos resistente.

Dentre as várias estratégias de manejo para evitar a resistência de plantas daninhas citadas na literatura destaca-se a utilização de herbicidas com diferentes mecanismos de ação em rotação, realização de aplicações sequenciais e uso de misturas de herbicidas com diferentes mecanismos de ação (Vidal e Merotto Junior, 2001). Entretanto, a atividade biológica do herbicida pós-emergente é afetada pelo estado morfológico da planta ao qual a mesma se encontra assim como as condições ambientais durante a aplicação (Gerber et al., 1983; Kudsk et al., 1990). Plantas sob estresse hídrico apresentam menores níveis de crescimento e podem produzir menores quantidades de clorofila (Botha e Botha, 1979). As plantas podem apresentar diversas alterações morfológicas em condições de estresse hídrico, entre elas a redução da condutância estomática (Caemmerer e Farquhar, 1981), redução da taxa fotossintética (Boyer, 1970) e aumento da produção de cera epicuticular (Skoss, 1955), dificultando assim controle pelo herbicida que está relacionado a alterações na retenção do herbicida, absorção, translocação ou metabolismo (Shahi, 1975). 
Nesse sentido, esse trabalho foi desenvolvido com os objetivos de avaliar a campo o melhor momento para iniciar o controle químico da buva (Conyza spp.) quando em condições de período seco, ou seja, de estresse hídrico e em condições de período úmido, considerada normais e adequadas a aplicação de herbicidas.

\section{Material e métodos}

\section{Descrição dos experimentos}

Os experimentos foram conduzidos no município de Indianópolis, no estado de Minas Gerais, no período de 18/09/2015 a 29/12/2015, sendo o clima predominante no município, de acordo com a classificação de Köppen, tipo Aw, tropical chuvoso com estação seca de inverno. Entre maio e outubro predominam as massas de ar Tropical Atlântica e Tropical Continental, gerando tempo bom, baixa nebulosidade e escassez de chuva na região (Borges, 2006). Os dados de precipitação dos dois experimentos durante a condução dos mesmos estão apresentados na Figura 1.

A área onde os experimentos foram instalados encontrava-se em pousio, sem cultura de inverno ou cobertura vegetal, o que propiciou a infestação de plantas de buva (Conyza spp.) em densidade média de 18 plantas $\mathrm{m}^{-2}$. A referida área experimental possui um histórico de mais de 5 anos de uso continuo de glifosato e constatando-se baixo nível de controle das plantas de buva quando empregado este herbicida. Os locais onde foram desenvolvidos os experimentos apresentaram as seguintes coordenadas: Latitude $18^{\circ} 58^{\prime}$ 02.29” S e Longitude $47^{\circ} 51^{\prime} 54.41^{\prime \prime} \mathrm{O}$, altitude 929 metros (Experimento 1) e Latitude $18^{\circ} 58^{\prime} 2.84^{\prime \prime} \mathrm{S}$ e Longitude $47^{\circ} 51^{\prime}$ '55.78'O, altitude 928 metros (Experimento 2), ambos apresentando solo de textura argilosa $(11 \%$ de areia, $2 \%$ de silte e $87 \%$ de argila), sob o delineamento experimental em blocos ao acaso e unidades experimentais constituídas por parcelas com dimensões de 5,0 m de largura por 6,0 $\mathrm{m}$ de comprimento $\left(30,0 \mathrm{~m}^{2}\right)$.

\section{Experimento 1}

O Experimento 1 foi realizado em esquema fatorial duplo, tendo como primeiro fator a comparação da condição climática do período onde realizou-se a primeira aplicação do herbicida glifosato + 2,4-D $(1.200+670$ g e.a. ha-1) As condições do período da aplicação inicial foram denominadas de período seco ou úmido, sendo período seco caracterizado pela ausência de chuva por mais de 30 dias na área utilizada, apresentando seca na camada superficial de 0-40 cm do solo. Já a aplicação referente ao período

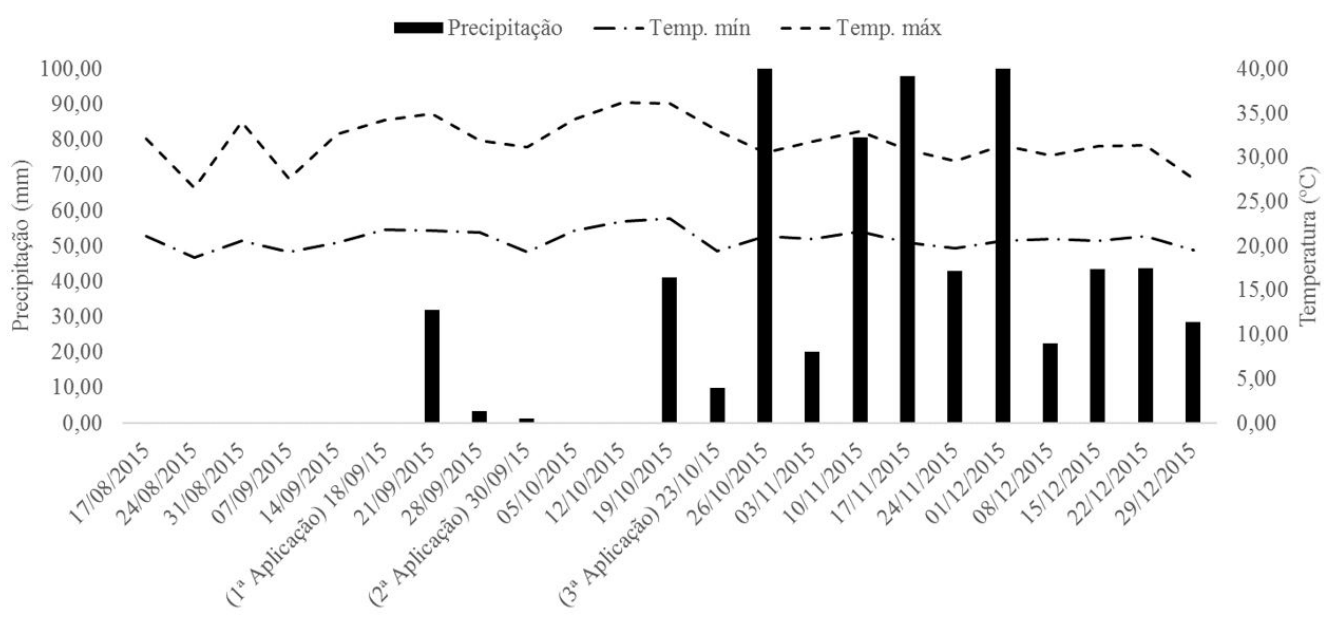

Figura 1. Dados de precipitação ao longo da condução dos experimentos em Indianópolis/MG, 2015. 
úmido, ocorreu chuva 12 dias após a aplicação do período seco, onde observou-se precipitação acumulada de mais de $35 \mathrm{~mm}$ (Figura 1). O segundo fator correspondeu da aplicação sequencial dos tratamentos herbicidas: glifosato (Roundup ${ }^{\circledR}$ Original, $480 \mathrm{~g} \mathrm{~L}^{-1}$ de e.a., SL, Monsanto) + 2,4-D (DMA ${ }^{\circledR} 806$ BR, 670 g L $^{-1}$ de e.a., SL, Dow AgroSciences) $\left(1.200+670\right.$ g e.a. ha $\left.{ }^{-1}\right)$, glifosato $+2,4-\mathrm{D}+$ diclosulam $\left(\right.$ Spider $^{\circledR} 840$ WG, $840 \mathrm{~g} \mathrm{Kg}^{-1}$ de i.a., WG, Dow AgroSciences) $\left(1.200\right.$ g e.a +670 g e.a. $+29,4$ g i.a. ha $\left.{ }^{-1}\right)$, diclosulam + paraquat (Gramoxone ${ }^{\circledR} 200,200 \mathrm{~g} \mathrm{~L}^{-1}$ de i.a., SL, Syngenta) $\left(29,4\right.$ g i.a. +500 g e.a. ha $\left.{ }^{-1}\right)$, paraquat $\left(500\right.$ g e.a. ha ${ }^{-1}$ ) e fluroxipir (Starane $200^{\circledR}, 200 \mathrm{~g} \mathrm{~L}^{-1}$ de e.a., EC, Dow AgroSciences) (200 g e.a. ha-1). Ainda, foi utilizado uma testemunha sem aplicação herbicida para efeito comparativo.

A primeira aplicação, considerada para o período seco, foi realizada no dia 18 de Setembro de 2015. Já a primeira aplicação, para o período úmido, ocorreu logo após as primeiras chuvas, realizada no dia 30 de Setembro de 2015. Mesmo com um intervalo de 12 dias entre as aplicações de período seco e úmido, as plantas de buva encontravam-se com altura variando entre 20 a $35 \mathrm{~cm}$, não apresentando diferenças de tamanhos médios da população de buva presente nas parcelas de um período para o outro. Uma segunda aplicação de herbicida realizada de forma sequencial para todos os tratamentos, foi realizada no dia 23 de Outubro de 2015, ou seja, 35 e 23 dias após a primeira aplicação para o período seco e úmido, respectivamente.

Para determinação da umidade presente no solo, no momento da primeira aplicação para os considerados períodos secos e úmidos, foram coletados quatro pontos dentro da área experimental de $0-40 \mathrm{~cm}$ de profundidade, com auxílio de trado cilíndrico manual, os quais foram retiradas sub-amostras de 150 gramas, conforme proposto por Reichardt (1985), seguindo após as mesmas colocadas em sacos de papel e levadas para estufa com circulação de ar forçada a $60^{\circ} \mathrm{C}$, por 72 horas, quando posteriormente foram pesadas as sub-amostras, quantificando-se a porcentagem de umidade no solo através do cálculo a seguir.

$$
\% \text { umidade solo }=\frac{\text { massa úmida }- \text { massa seca }}{\text { massa seca }} \times 100
$$

Para o solo coletado no período seco, a umidade média foi de $1,4 \%$ e, no período úmido, foi constatado média de 40,2\%. Vale ressaltar que além do solo do período úmido apresentar umidade na camada de $0-40 \mathrm{~cm}$ superior ao do período seco, foi possível notar que as plantas de buva apresentavam-se com maior vigor no momento da aplicação.

Para todas as aplicações herbicidas foi utilizado pulverizador costal de pressão constante à base de $\mathrm{CO}_{2}$, equipado com barra de seis pontas tipo leque, modelo AIXR110.015, sob pressão de $2,0 \mathrm{kgf} \mathrm{cm}^{-2}$, que proporcionou volume de calda equivalente à $100 \mathrm{~L} \mathrm{ha}^{-1}$. As condições climáticas no momento das aplicações eram: umidade relativa do ar de 50, 58 e 57\%; temperatura do ar de $32,29,28^{\circ} \mathrm{C}$; velocidade do vento de $1,6,2,1$ e $2,8 \mathrm{~km} \mathrm{~h}^{-1}$; temperatura do solo de 24,23 e $21^{\circ} \mathrm{C}$; para a primeira, segunda e terceira aplicação, respectivamente.

\section{Experimento 2}

No Experimento 2, visando verificar o nível de dificuldade de controle da buva ao herbicida glifosato presente na área experimental de ambos experimentos, foram testadas, em aplicações sequenciais, doses crescentes 0, 480, 960, 1.920 e 3.840 g e.a. ha ${ }^{-1}$ de glifosato, bem como 2,4-D a $670 \mathrm{~g}$ e.a. ha-1 e a associação de glifosato $+2,4-\mathrm{D}(960+670$ g e.a. ha-1 $)$.

As aplicações dos tratamentos herbicidas ocorreram no dia 18 de Setembro de 2015, momento esse em que o clima encontrava-se com características de período seco, conforme Figura 1, e as plantas de buva apresentavam-se com altura de 20 a $35 \mathrm{~cm}$. Após 35 dias da primeira aplicação, no dia 23 de Outubro de 2015, foi realizado uma segunda 
aplicação com os mesmos tratamentos, sendo que neste momento as plantas de buva encontravam-se com altura média de $40 \mathrm{~cm}$. As aplicações seguiram os mesmos parâmetros apresentados no Experimento 1. As condições climáticas no momento das aplicações eram: umidade relativa do ar de 50 e $59 \%$; temperatura do ar de 32 e $29^{\circ} \mathrm{C}$; velocidade do vento de 1,6 e $2,0 \mathrm{~km} \mathrm{~h}^{-1}$; temperatura do solo de 24 e $23^{\circ} \mathrm{C}$, para a primeira e segunda aplicação, respectivamente.

\section{Parâmetros avaliados}

Em ambos experimentos foi avaliado a porcentagem de controle, com base em escala visual de notas, onde $0 \%$ correspondeu à "ausência de eficácia de controle" e 100\% correspondeu à “eficácia de controle total” (Velini, 1995).

As avaliações de controle para o Experimento 1 foram realizadas aos 14, 28, 35 e 42 dias após a aplicação (DAA) sequencial. No Experimento 2 , foi avaliado a porcentagem de controle aos 15 e 30 dias após cada aplicação, sendo que na última avaliação, referente a segunda aplicação, foram coletados a parte aérea das plantas de buva remanescentes nas parcelas para obtenção da massa seca. As mesmas foram coletadas, em duas partes distintas da parcela composta por uma área de $0,5 \mathrm{~m}^{2}$, resultando em $1 \mathrm{~m}^{2}$, as quais foram armazenadas em saco de papel e levados para estufa com circulação de ar forçada a $60^{\circ} \mathrm{C}$ até atingir peso constante.

Como a testemunha é referência visual para a atribuição do efeito dos tratamentos, e possuem valores fixos (0 ou 100\%), optou-se pela não inclusão desta na análise estatística de eficácia de controle, uma vez que os experimentos visavam a comparação dos tratamentos herbicidas.

Os dados de controle e massa seca foram submetidos a análise de variância (ANOVA) pelo teste $\mathrm{F}$, e as médias, quando significativas, comparadas pelo teste de Tukey, a 5\% de probabilidade.

\section{Resultados e discussão}

Os resultados obtidos de controle de buva, aplicada nos diferentes períodos climáticos no Experimento 1, podem ser observados na Tabela 1. Foi notado que o fator período climático no momento da primeira aplicação não influenciou na eficiência individual dos produtos, ou seja, os resultados não apresentaram diferença estatística entre a aplicação realizada no período seco ou úmido, independente do momento de avaliação ou herbicida utilizado.

$\mathrm{Na}$ literatura existem relatos que a condição hídrica do solo pode ou não influenciar o controle de plantas daninhas, como exposto por Zhang et al. (2001) que o herbicida imazethapyr aplicado em pós-emergência de Oryza sativa não foi influenciado pela condição hídrica, entretanto Roman et al. (2005) demonstraram que Euphorbia heterophylla submetidas a aplicação de carfentrazone-ethyl apresentaram melhores controles quando estavam sem déficit hídrico. Esse fato, provavelmente, se deve ao mecanismo de ação de cada herbicida citado.

Ainda na Tabela 1, pode-se observar que os tratamentos sequenciais com o herbicida diclosulam na composição, favoreceram a velocidade de controle, em especial quando associado ao Paraquat, onde níveis de controle numericamente superiores a $90 \%$ foram observados aos 14 DAA, para aplicações iniciadas no período seco ou úmido. Já o herbicida diclosulam associado ao glifosato + 2,4-D proporcionou controle superior à $90 \%$ aos 14 DAA somente para aplicação iniciada em período seco. Dentre os herbicidas utilizados na sequencial, o fluroxipir apresentou a menor velocidade de controle aos 14 DAA, especialmente quando aplicado em período seco, sendo estatisticamente inferior as misturas com 2,4-D e, ou diclosulam e numericamente inferior ao paraquat quando utilizado de forma isolada. Vale destacar que essas diferenças entre tratamentos foram menores no mesmo período (14 DAA) quando as aplicações iniciaram no período úmido, 
Tabela 1. Porcentagem visual de controle dos tratamentos herbicidas após a aplicação sequencial ao tratamento de glifosato $+2,4-\mathrm{D}\left(1.200+670 \mathrm{~g}\right.$ e.a. ha $\left.^{-1}\right)$ realizada na condição período seco e úmido. Indianópolis/ MG, 2015.

\begin{tabular}{|c|c|c|c|c|c|c|c|}
\hline \multirow[b]{2}{*}{$\begin{array}{l}\text { Condição do período } \\
\text { na primeira aplicação e } \\
\left.\text { tratamento (g e.a. ha }{ }^{-1}\right)\end{array}$} & \multicolumn{5}{|c|}{ Tratamentos herbicidas aplicados na sequencial (g e.a. ou i.a. ha-1) } & \multirow{2}{*}{\multicolumn{2}{|c|}{ DMS }} \\
\hline & $\begin{array}{c}\text { glifosato + 2,4-D } \\
(1.200+670)\end{array}$ & $\begin{array}{c}\text { glifosato + 2,4-D + } \\
\text { diclosulam } \\
(1.200+670+29,4)\end{array}$ & $\begin{array}{l}\text { paraquat }+ \\
\text { diclosulam } \\
(500+29,4)\end{array}$ & $\begin{array}{l}\text { paraquat } \\
(500)\end{array}$ & $\begin{array}{l}\text { fluroxipir } \\
\text { (200) }\end{array}$ & & \\
\hline \multicolumn{8}{|c|}{14 DAA } \\
\hline glifosato & $88,3 \mathrm{~A} \mathrm{a}$ & $91,8 \mathrm{Aa}$ & $92,8 \mathrm{Aa}$ & $83,5 \mathrm{~A}$ ab & $74,3 \mathrm{Ab}$ & 10,53 & 5,34 \\
\hline $\begin{array}{ll}\text { Úmido } & +2,4-\mathrm{D} \\
& (1.200+670)\end{array}$ & $89,5 \mathrm{Aa}$ & $89,0 \mathrm{Aab}$ & $92,0 \mathrm{Aa}$ & $80,0 \mathrm{~A} \mathrm{ab}$ & $85,0 \mathrm{~A}$ ab & 13,05 & 6,67 \\
\hline DMS & 7,94 & 9,67 & 4,18 & 8,67 & 17,06 & & \\
\hline CV & 3,97 & 4,75 & 2,01 & 4,71 & 10,12 & & \\
\hline \multicolumn{8}{|c|}{28 DAA } \\
\hline glifosato & $98,8 \mathrm{Aa}$ & $100,0 \mathrm{Aa}$ & $97,0 \mathrm{Aa}$ & $94,3 \mathrm{Aa}$ & $97,3 \mathrm{~A} \mathrm{a}$ & 7,42 & 3,33 \\
\hline $\begin{array}{ll}\text { Úmido } & +2,4-\mathrm{D} \\
& (\mathbf{1 . 2 0 0}+\mathbf{6 7 0 )} \\
\end{array}$ & $99,5 \mathrm{~A} \mathrm{a}$ & $98,7 \mathrm{Aa}$ & $99,3 \mathrm{~A} \mathrm{a}$ & 91,9 A a & $91,1 \mathrm{~A} \mathrm{a}$ & 8,85 & 8,58 \\
\hline DMS & 10,36 & 5,79 & 9,44 & 5,77 & 7,79 & & \\
\hline CV & 8,15 & 7,14 & 7,3 & 4,58 & 6,41 & & \\
\hline \multicolumn{8}{|c|}{ 35 DAA } \\
\hline glifosato & $98,5 \mathrm{Ac}$ & $100,0 \mathrm{Aa}$ & $96,8 \mathrm{~A} \mathrm{ab}$ & $94,8 \mathrm{Ac}$ & $96,8 \mathrm{~A} \mathrm{bc}$ & 7,87 & 3,55 \\
\hline $\begin{array}{ll}\text { Úmido } & +2,4-\mathrm{D} \\
& (1.200+670)\end{array}$ & $99,5 \mathrm{~A} \mathrm{a}$ & $98,1 \mathrm{Aa}$ & $99,3 \mathrm{~A} \mathrm{a}$ & $92,2 \mathrm{~A} \mathrm{a}$ & $90,8 \mathrm{~A} \mathrm{a}$ & 9,11 & 10,9 \\
\hline DMS & 11,66 & 6,56 & 10,2 & 5,69 & 6,85 & & \\
\hline $\mathrm{CV}$ & 8,63 & 7,66 & 7,7 & 4,65 & 5,26 & & \\
\hline \multicolumn{8}{|c|}{ 42 DAA } \\
\hline glifosato & $100,0 \mathrm{Aa}$ & $99,5 \mathrm{Aa}$ & $96,8 \mathrm{~A} \mathrm{a}$ & $97,8 \mathrm{Aa}$ & $97,5 \mathrm{Aa}$ & 11,4 & 5,11 \\
\hline $\begin{array}{ll}\text { Úmido } & +2,4-D \\
& (\mathbf{1 . 2 0 0}+\mathbf{6 7 0 )} \\
\end{array}$ & $98,7 \mathrm{Aa}$ & $99,0 \mathrm{~A} \mathrm{a}$ & $99,9 \mathrm{~A} \mathrm{a}$ & $94,3 \mathrm{~A} \mathrm{a}$ & $93,8 \mathrm{~A} \mathrm{a}$ & 14,27 & 10,95 \\
\hline DMS & 5,79 & 5,48 & 3,62 & 18,81 & 14,24 & & \\
\hline $\mathrm{CV}$ & 7,14 & 6,02 & 4,74 & 11,95 & 9,99 & & \\
\hline
\end{tabular}

g e.a. = gramas de equivalente ácido; g i.a. = gramas de ingrediente ativo; DAA = Dias Após Aplicação Sequencial. CV (\%): Coeficiente de variação. Valores seguidos de letras iguais maiúsculas na mesma coluna e minúsculas na mesma linha não diferem estatisticamente entre si pelo teste de Tukey $(\mathrm{p}<0,05)$.

e desapareceram à medida em que as avaliações avançaram, constatando-se ausência de diferença entre os tratamentos na avaliação final de controle (42 DAA), independente da condição de umidade na aplicação inicial.

De acordo com Constantin et al. (2013), plantas de buva em estádios avançados de desenvolvimento, acima de $20 \mathrm{~cm}$, tratadas com aplicação única de herbicidas, muitas vezes não são controladas de maneira eficiente. Para estes casos é recomendada a aplicação sequencial de herbicidas sistêmicos na primeira aplicação seguido de aplicação de herbicida de contato aos
7 a 15 dias após a primeira aplicação. De maneira geral, este sistema de manejo acelera a velocidade inicial e final de controle desta espécie. Assim, esses resultados corroboram à observação de Constantin et al. (2013), pois a aplicação de glifosato $+2,4-\mathrm{D}$ em ambos os períodos (seco ou úmido), seguido pela aplicação sequencial de herbicida de contato apresentou aceitável controle de buva, desde os 14 até 42 DAA. Ressalta-se que quando o herbicida diclosulam foi associado ao paraquat, os níveis de controle da buva foram numericamente superiores aos demais, especialmente na primeira avaliação. 
Tabela 2. Porcentagem visual de controle realizada aos 15 e 30 dias após cada aplicação dos tratamentos contendo diferentes doses do glifosato e massa seca das plantas de buva. Indianópolis/ MG, 2015.

\begin{tabular}{|c|c|c|c|c|c|c|c|c|c|c|c|c|}
\hline \multicolumn{2}{|r|}{ Tratamentos } & $\begin{array}{c}\text { Dose } \\
\text { g e.a. } \text { ha }^{-1}\end{array}$ & \multicolumn{2}{|c|}{$\begin{array}{l}15 \text { DAA } \\
1^{\mathrm{a}} \text {. Apl. }\end{array}$} & \multicolumn{2}{|c|}{$\begin{array}{l}30 \text { DAA } \\
1^{\text {a }} \text {. Apl. }\end{array}$} & \multicolumn{2}{|c|}{$\begin{array}{l}15 \text { DAA } \\
2^{\mathrm{a}} \text {. Apl. }\end{array}$} & \multicolumn{2}{|c|}{$\begin{array}{l}30 \text { DAA } \\
2^{\mathrm{a}} \text {. Apl. }\end{array}$} & \multicolumn{2}{|c|}{$\begin{array}{c}\text { Massa Seca } \\
\text { (gramas) }\end{array}$} \\
\hline 1 & Testemunha & & 0,0 & & 0,0 & & 0,0 & & 0,0 & & 184,62 & \\
\hline 2 & glifosato & 480 & 5,3 & $\mathrm{c}$ & 27,5 & $\mathrm{c}$ & 35,0 & $\mathrm{e}$ & 37,5 & $\mathrm{e}$ & 127,80 & $\mathrm{~b}$ \\
\hline 3 & glifosato & 960 & 10,0 & $\mathrm{bc}$ & 55,0 & $\mathrm{~b}$ & 48,8 & d & 48,8 & $d$ & 107,02 & $\mathrm{~b}$ \\
\hline 4 & glifosato & 1.920 & 4,8 & $\mathrm{c}$ & 60,0 & $\mathrm{~b}$ & 60,8 & $\mathrm{c}$ & 60,0 & $\mathrm{c}$ & 113,40 & $\mathrm{~b}$ \\
\hline 5 & glifosato & 3.840 & 3,8 & $\mathrm{c}$ & 60,0 & $\mathrm{~b}$ & 64,3 & $\mathrm{c}$ & 63,5 & $\mathrm{c}$ & 126,82 & $b$ \\
\hline 6 & 2,4-D & 670 & 15,0 & $\mathrm{~b}$ & 73,8 & $\mathrm{a}$ & 82,5 & $\mathrm{~b}$ & 82,0 & $\mathrm{~b}$ & 94,46 & $\mathrm{bc}$ \\
\hline 7 & glifosato $+2,4-\mathrm{D}$ & $960+670$ & 28,8 & $\mathrm{a}$ & 78,5 & $\mathrm{a}$ & 94,3 & $\mathrm{a}$ & 94,5 & $\mathrm{a}$ & 46,69 & $\mathrm{c}$ \\
\hline & CV $(\%)$ & & \multicolumn{2}{|c|}{34,97} & \multicolumn{2}{|c|}{7,57} & \multicolumn{2}{|c|}{4,78} & \multicolumn{2}{|c|}{6,46} & \multicolumn{2}{|c|}{20,32} \\
\hline
\end{tabular}

Apl. = Aplicação; g e.a. = gramas de equivalente ácido; DAA = Dias Após a Aplicação. CV (\%): Coeficiente de variação. Valores seguidos de letras iguais na mesma coluna não diferem estatisticamente entre si pelo teste de Tukey $(\mathrm{p}<0,05)$.

No Experimento 2, foi possível identificar que a população de buva presente na área experimental apresentou baixo nível de controle pelo glifosato, proporcionado pela dose recomendada em bula (960 g e.a. ha ${ }^{-1}$ ) menos de 50\% (Tabela 2), mesmo com aplicação sequencial deste herbicida e, ou incremento de dose em até quatro e oito vezes, totalizando 1.920 ou 3.840 g e.a. ha-1, apresentando controles não superiores à $60,0 \mathrm{ou}$ $63,5 \%$, respectivamente. Assim, essa informação respalda a necessidade de adoção de manejo e, ou aplicações sequenciais, como apresentado no Experimento 1, uma vez que o glifosato apresenta baixa eficiência sobre esta população local.

Levando-se em conta somente o conceito de resistência agronômico, que determina que para classificar uma planta como resistente seja necessário que a população sobreviva à dose recomendada do herbicida em condições normais de campo, pode-se inferir que esta população de buva na área de condução do experimento é resistente ao glifosato (Christoffoleti e Nicolai, 2016). Porém, para Heap (2017a) existe um problema com o uso apenas da definição agronômica, pois a mesma pode variar de região para região, cultura em que o produto é aplicado e ainda questões econômicas que determinam a dose, o que poderia classificar a mesma população de uma espécie como suscetível em uma cultura e resistente na outra.

É possível observar na Tabela 2 que o controle desta espécie foi superior com o uso do herbicida 2,4-D na dose de $670 \mathrm{~g}$ e.a. ha ${ }^{-1}$, proporcionando níveis acima de $70 \%$ ou $80 \%$, com uma ou duas aplicações, respectivamente, mesmo quando esta população de buva não foi controlada com glifosato em doses até oito vezes a recomendada (3.840 g e.a. ha-1). Por fim, resultados superiores e aceitáveis foram proporcionados pela aplicação sequencial de glifosato $+2,4-\mathrm{D}\left(960+670\right.$ g e.a. ha $\left.{ }^{-1}\right)$ com níveis acima de $94 \%$, que consequentemente resultou nos menores valores médios de massa seca das plantas de buva, dentre os tratamentos. Segundo Takano et al. (2013), a adição de 2,4-D ao glifosato pode levar a um efeito sinérgico no controle de buva nos estádios de desenvolvimento (menores que $6 \mathrm{~cm}, 6$ a $15 \mathrm{~cm}$ e maiores que $15 \mathrm{~cm}$ ), sendo importante ressaltar que somente no estádio de até $6 \mathrm{~cm}$ de altura a mistura glifosato $+2,4-\mathrm{D}$ $\left(720+670\right.$ g e.a. ha $\left.{ }^{-1}\right)$ foi eficaz no controle de Conyza spp. (85\%).

De forma geral, a população de buva demonstrou não ser controlada somente com o herbicida glifosato, o qual não proporciona controle aceitável mesmo em doses superiores a indicada na bula. Além disso, para as populações 
de buva que apresentam nível alto de dificuldade de controle pelo glifosato, é necessário realizar manejo sequencial de herbicidas para alcançar resultados superiores e, ou aceitáveis, sendo que a aplicação inicial de glifosato $+2,4-\mathrm{D}$, em período seco ou úmido, seguido da aplicação de glifosato +2,4-D ou paraquat, ambos associados ou não ao diclosulam, ou fluroxipir isolado, proporcionaram controle final satisfatório permitindo a condução normal da lavoura, ao exemplo da soja.

Vale adicionar, que mesmo as aplicações sequenciais tenham proporcionado controles satisfatórios da buva, uma das estratégias para mitigar o avanço dos casos de resistência é a diversificação no uso de herbicidas, como rotação de mecanismos de ação e uso de pré e pós-emergentes, quando possível (Christoffoleti e Nicolai, 2016).

\section{Conclusões}

O fator período climático (períodos secos ou úmidos) no momento da primeira aplicação não influencia na eficiência individual dos produtos.

A aplicação inicial de glifosato + 2,4-D, em período seco ou úmido, seguido da aplicação de glifosato $+2,4-\mathrm{D}$ ou paraquat, ambos associados ou não ao diclosulam, ou fluroxipir isolado, proporcionam controle final satisfatório.

É necessário realizar manejo sequencial de herbicidas para alcançar resultados superiores e, ou aceitáveis em populações de buva que não apresentem controle com a aplicação de dose recomendada de glifosato.

\section{Referências}

Borges, A.A.S. Diagnóstico ambiental e proposta de monitoramento da área do lago de Miranda pertencente ao município de Indianópolis-MG. 2006. 141 f. Dissertação (Mestrado em Geografia) - Instituto de Geografia, Universidade Federal de Uberlândia, Uberlândia, 2006.
Botha, F.C.; Botha, P.J. The effect of water stress on the nitrogen metabolism of two maize lines: II. Effects on the rate of protein synthesis and chlorophyll content. Zeitschrift für Pflanzenphysiologie, v.94, n.2, p.179-183, 1979.

Boyer, J.S. Leaf enlargement and metabolic rates in corn, soybean and sunflower at various leaf water potentials. Plant Physiology, v.46, n.2, p.233-235, 1970.

Caemmerer, S.V.; Farquhar, G.D. Some relationships between the biochemistry of photosynthesis and the gas exchange of leaves. Planta, v.153, n.4, p.376-387, 1981.

Christoffoleti, P.J.; Nicolai, M. Aspectos de resistência de plantas daninhas a herbicidas. 4.ed. Piracicaba: Associação Brasileira de Ação a Resistência de Plantas Daninhas - HRAC-BR, 2016. cap. 1. p.11-31.

Cici, S.Z.H.; Van Acker, R.C. A review of the recruitment biology of winter annual weeds in Canada. Canadian Journal of Plant Science, v.89, n.3, p.575-589, 2009.

Constantin, J.; Oliveira Júnior, R.S.; Oliveira Neto, A.M. Buva: fundamentos e recomendações para manejo. Curitiba: Omnipax, 2013. 104 p.

Gerber, H.R.; Nyffeler, A.D.H.; Green, T.D. The influence of rainfall, temperature, humidity and light on soil and foliage-applied herbicides. Aspects of Applied Biology, v.4, p.1-14, 1983.

Green, T.D. The ecology of fleabane (Conyza spp.). $173 \mathrm{f}$. Thesis (Doctor of Philosophy) - School of Environmental and Rural Science, University of New England, New England, 2010.

Heap, I.A. Criteria for confirmation of the herbicide-resistant weeds. Disponível em: $<$ http:// www.weedscience.org > . Acesso em: 31 jul. 2017a.

Heap, I.A. International survey of herbicideresistant weeds. Disponível em: <http://www. weedscience.org > . Acessado em: 20 fev. $2017 \mathrm{~b}$. 
Kudsk, P.; Olesen, T.; Thonke, K.E. The influence of temperature, humidity and simulated rain on the performance of thiameturon-methyl. Weed Research, v.30, n.4, p.261-269, 1990.

Marochio, C.A.; Bevilaqua, M.R.R.; Takano, H.K.; Mangolim, C. A.; Oliveira Junior, R.S.; Silva Machado, M.F.P.S. Genetic admixture in species of Conyza (Asteraceae) as revealed by microsatellite markers. Acta Scientiarum, Agronomy, v.39, n.4, p.437-445, oct.-dec. 2017.

Moreira, M.S.; et al. Resistência de Conyza Canadensis e C. bonariensis ao herbicida glyphosate. Planta Daninha, v.25, n.1, p.157-164, 2007.

Reichardt, K. Processos de transferência no sistema solo-planta-atmosfera. 4.ed. Campinas: Fundação Cargill, 1985. 445p.

Roman, E.S.; Vargas, L.; Ribeiro, M.C.F. Efeito do teor de umidade do solo na seletividade e na eficácia de carfentrazone-ethyl no controle de plantas daninhas na cultura da soja. Revista Brasileira de Herbicidas, v.4, n.2, p.114-122, 2005.

Santos, G.; Oliveira Junior, R.S.; Constantin, J.; Francischini, A.C.; Osipe, J.B. Multiple resistance of Conyza sumatrensis to Chlorimuronethyl and to glyphosate. Planta Daninha, v.32, n.2, p.409416, 2014.

Shahi, H.N. Effect of soil moisture stress on the absorption, translocation, and herbicidal efficiency of foliage-applied herbicides. International Pest Control, v.17, p.13-17, 1975.

Shrestha, A.; Hanson, B.D.; Fidelibus, M.W.; Alcorta, M. Growth, phenology, and intraspecific competition between glyphosate-resistant and glyphosate-susceptible horseweeds (Conyza canadensis) in the San Joaquin Valley of California. Weed Science, v.58, n.2, p.147-153, 2010.

Skoss, J.D. Structure and composition of plant cuticle in relation to environmental factors and permeability. Botanical Gazette, v.117, n.1, p.55-72, 1955.

Takano, H.K.; Oliveira Junior, R.S.; Constantin, J.; Biffe, D.F.; Franchini, L.H.M.; Braz, G.B.P.; et al. Efeito da adição do 2,4-D ao glyphosate para o controle de espécies de plantas daninhas de difícil controle. Revista Brasileira de Herbicidas, v.12, n.1, p.1-13, 2013.

Theabaud, C.; Abbott, R.J. Characterization of invasive Conyza species (Asteraceae) in Europe: Quantitative trait and isozyme analysis. American Journal of Botany, v.82, n.3, p.360-368, 1995.

Tozzi, E.; Van Acker, R.C. Effects of seedling emergence timing on the population dynamics of horseweed (Conyza canadensis var. canadensis). Weed Science, v.62, n.3, p.451-456, 2014.

Vargas, L.; Bianchi, M.A.; Rizzardi, M.A.; Agostinetto, D.; Dal Magro, T. Buva (Conyza bonariensis) resistente ao glyphosate na região sul do Brasil. Planta Daninha, v.25, n.3, p.573578, 2007.

Velini, E.D. Estudo e desenvolvimento de métodos experimentais e amostrais adaptados à matologia. 1995. 250f. Tese (Doutorado) Universidade Estadual Paulista de Jaboticabal, Jaboticabal, 1995.

Vidal, R.A.; Merotto Junior, A. Herbicidologia. Porto Alegre: Edição dos Autores, 2001. 152p.

Zhang, W.; Webster, E.P.; Selim, H.M. Effect of soil moisture on efficacy of imazethapyr in greenhouse. Weed Technology, v.15, n.2, p.355-359, 2001. 